\title{
Childhood Intraocular Retinoblastoma
}

National Cancer Institute

\section{Source}

National Cancer Institute. Childhood Intraocular Retinoblastoma. NCI Thesaurus. Code C9047.

Retinoblastoma during childhood that has not spread beyond the eye. 